\title{
Development of a puff- and suction-type pressure stimulator for human tactile studies
}

\author{
Hyung-Sik Kim ${ }^{1} \cdot$ Mi-Hyun Choi ${ }^{1} \cdot$ Soon-Cheol Chung ${ }^{1}$
}

Published online: 14 April 2017

(C) Psychonomic Society, Inc. 2017

\begin{abstract}
In this study, we developed a tactile stimulator capable of administering either puff- or suction-type stimuli. The system is composed of three parts: a control unit, an airhandling unit, and a stimulation unit. The control unit controls the type, intensity, and time of stimulation. The air-handling unit delivers the stimulation power quantitatively to the stimulation unit, as commanded by the control unit. The stimulation unit stably administers either type of pressure to the skin, without any change of the tactor. Although the design of the stimulator is simple, it allows for five levels of control of the stimulation intensity (2-6 psi) and 0.1-s steps of control of the stimulation time, as we confirmed by tests. Preliminary electroencephalographic and event-related potential (ERP) studies of our system in humans confirmed the presence of N100 and P300 waves at standard electrode position C3, which are related to perception and cognition, respectively, in the somatosensory area of the brain. In addition, different stimulation types (puff and suction) and intensities (2 and 6 psi) were reflected in different peak-to-peak amplitudes and slopes of the mean ERP signal. The system developed in this study is expected to contribute to human tactile studies by providing the ability to administer puff- or suction-type stimuli interchangeably.
\end{abstract}

Keywords Tactile stimulator $\cdot$ Pressure sense $\cdot$ Puff $\cdot$ Suction

Soon-Cheol Chung

scchung@kku.ac.kr

1 Department of Biomedical Engineering, BK21+ Research Institute of Biomedical Engineering, College of Science \& Technology, Konkuk University, 268 Chungwon-daero, Chungju-si, Chungbuk 27478, South Korea
Currently, in the fields of brain and cognitive science (Allen \& Humphreys, 2009; Dresel et al., 2008; Jousmaki, Nishitani, \& Hari, 2007), neurophysiology (Golaszewski et al., 2002; Skedung et al., 2013), ergonomics (Auvray, Gallace, Hartcher-O'Brien, Tan, \& Spence, 2008; Gallace et al. 2007; Ho, Santangelo, \& Spence, 2009), clinical skill acquisition, and surgery simulation (Bark et al., 2013; King et al., 2008; Philip et al., 2015), many studies have been conducted on the tactile sense. Because the necessity of providing an interface for tactile information in virtual or actual environments is increasing, various devices for delivering tactile stimulation have been developed (Gwilliam, Bianchi, Su, \& Okamura, 2013; Monnai et al., 2014; Suzuki \& Kobayashi, 2005). This is usually followed by electroencephalographic (EEG), functional magnetic resonance imaging (fMRI), or psychological studies to evaluate the performance and utility of each device (Ritter, Moosmann, \& Villringer, 2009; Yuan et al., 2010). Mechanical (Gallasch et al., 2010; Gwilliam et al., 2013; Wall \& Brewster, 2006), electrical (Echenique \& Graffigna, 2011; Kaczmarek et al., 2006; Yamamoto, Nagasawa, Yamamoto, \& Higuchi, 2006), and thermal methods (Chouvardas, Miliou, \& Hatalis, 2008; Kim et al., 2014; Yang, Wang, \& Chen, 2015) for providing the three submodalities of tactile sensation (i.e., vibrotactile, pressure, and thermal) are being used.

Among the three submodalities, the pressure sense distinguishes the degree of pressure applied to the surface of the skin, and is generally stimulated by mechanical probes such as pins, sticks, or filaments. Using this approach, a finger sized down to a $<1-\mathrm{mm}$ point surface is applied to stimulate the pressure sense, or an array of multiple pins is arranged to deliver to the user information such as texture, text, or direction (Chouvardas et al., 2008). Recently, a new method using an air-spray approach (also known as a pneumatic air jet) puff was developed to provide a pressure sensation. This 
technology is promising for utilization in a natural user interface (NUI), and is capable of providing stimulation without direct contact between the actuator and the skin (Gwilliam et al., 2013; Tsalamlal, Ouarti, \& Ammi, 2013). Using the air-spray method, the weight of an object can be applied to the object's virtual footprint on the skin, or a virtual texture, shape, or compliance can be provided (Chouvardas et al., 2008; Gwilliam et al., 2013; Monnai et al., 2014; Suzuki \& Kobayashi, 2005; Tsalamlal et al., 2013). Recently, a study was conducted to implement the edges or sharpness of a corner by a tactile illusion that uses suction, which has a direction opposite to that of an air spray (puff; Makino \& Shinoda, 2005; Porquis, Konyo, \& Tadokoro, 2012). The suction is generated by drawing air through an air tube or pipe and is classified as pressure-sense stimulation because it causes a skin deformation.

A comparative study on these two types of stimulation (puff and suction) using air pressure was conducted at the single-cell level (Bagriantsev, Gracheva, \& Gallagher, 2014; Martinac, 2004), but no comparative study at the human skin level has been conducted so far. To implement various and realistic tactile senses in virtual or actual environments with the aim of realizing NUIs, mechanistic investigations of both types of pressure at the human skin level are essential.

For this, the development of tactile simulators capable of quantitatively providing the two types of pressure stimulation is essential. Therefore, in this study, an air compressor and valve were used to provide both types of pressure with a single tactor, which eventually led to the development of a puff and suction stimulator capable of quantitatively controlling the stimulation parameters using a microprocessor. We test the performance of the developed system, and collect preliminary electroencephalogram (EEG) data from 20 human participants to verify the applicability of the system.

\section{Method}

The developed pressure stimulation system is shown in Fig. 1. The system is composed of three parts: a control unit, airhandling unit, and stimulation unit. The system can be controlled manually or by a PC running E-Prime software (Psychology Software Tools, Inc., USA), which is often used for visual and audio stimulation in the fields of recognition science and psychology.

\section{Control unit}

In the control unit, the type of stimulation (suction or puff), intensity of stimulation (five levels), and stimulation time (variable in steps of $0.1 \mathrm{~s}$ ) are controlled. The main microcontroller uses an ATmega128 (Atmel, USA), which is an eightbit AVR-based microcontroller. The controller has 53 generalpurpose input-output ports (GPIOs), a ten-bit analog-todigital converter, two eight-bit and 16-bit timer/counters, and a 16-MHz clock speed.

The type of stimulation is suction or puff and can be selected by individual ON/OFF control of Valve 1 (model HDW022T-3) and Valve 2 (model HDW022G-3), which are three-way solenoid valves (KCC Precision Co. Ltd., South

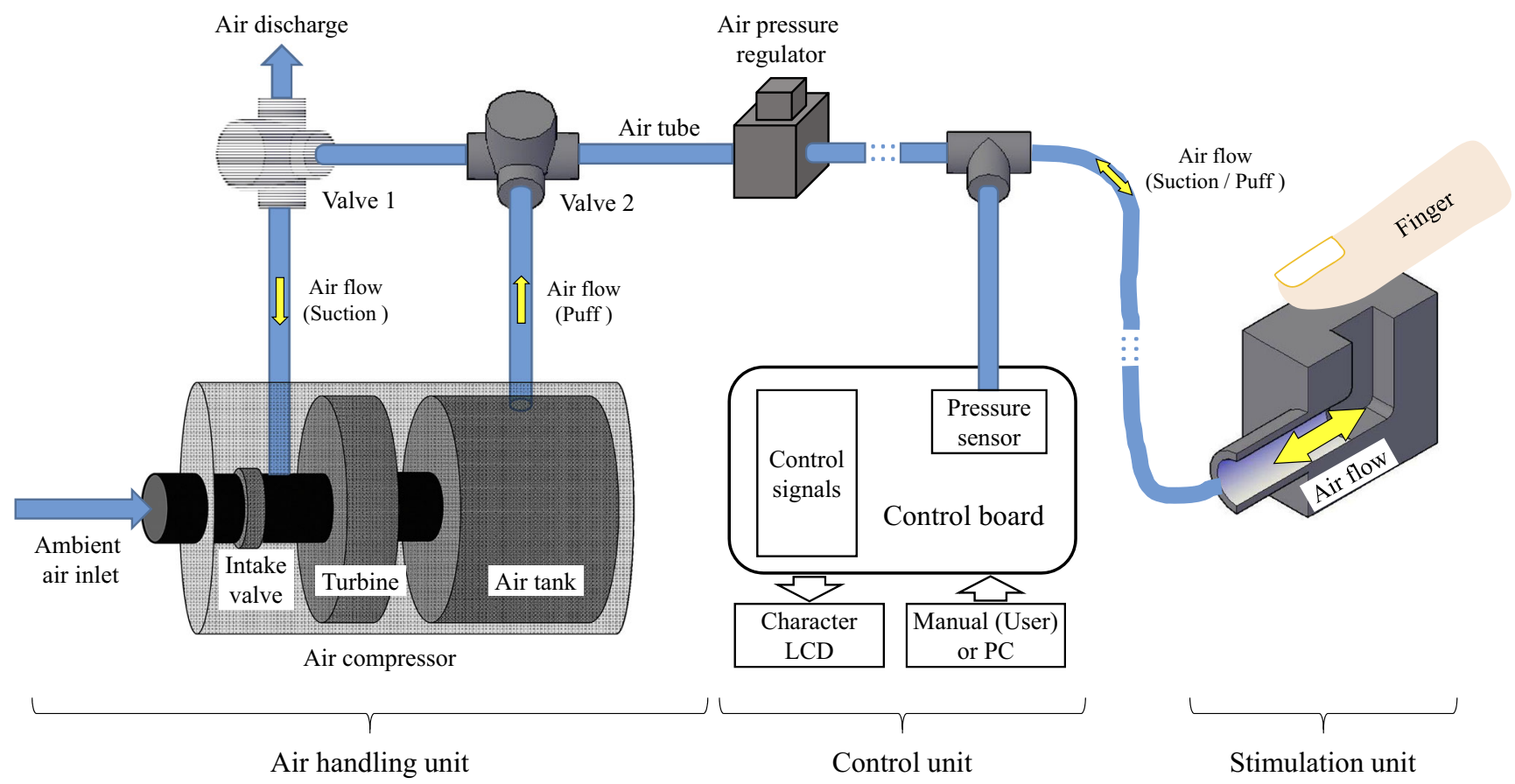

Fig. 1 Overall configuration of the developed puff- and suction-type pressure stimulation system 
Korea). By defining four operational modes via the software in advance, the user can create two types of pressure stimulation using a single air compressor (Table 1). In suctionstimulus mode, Valve 1, Valve 2, and the intake valve are controlled to make the air only intake from the stimulation unit, in order to create pressure of the suction type. The intake of air is powered by a turbine inside the air compressor. After every tactile stimulation of the suction type is displayed, the inside of the air tube is under residual negative pressure. To prepare for the next trial, the air-discharge mode is entered to return the system to its original status, and air is discharged from the port not connected to the air tube of Valve 1 to make the pressure inside the air tube atmospheric. In puff stimulus mode, the compressed air stored in the air tank is delivered to the stimulation unit to generate puff-type pressure. During air intake mode, the compressed air from the air tank consumed by puff stimulation is replaced.

The intensity of stimulation is controlled to one of five levels, ranging in steps of 1 psi from 2 to 6 psi, by controlling the flow rate of the compressed air that passes through the air tube. The flow rate is proportional to the cross-section where the air is controlled; thus, a custom-made air pressure regulator is placed in the middle of the air tube to alter the cross section. The pressure of the compressed air downstream from the pressure regulator is measured and digitized by pressure sensors 33A-005G-2210 (Smate, Taiwan) and SPSB-V01-M5 (TPC Mechatronics, South Korea). These sensors are positioned close to the stimulation unit, and monitoring was conducted in real time. From these data, the stimulation intensity could be electronically controlled and regulated. In addition, these data is used to correct errors when measuring brain signals. These errors are caused by the onset delay that takes place when the compressed air is conveyed to the stimulation unit through an air tube - in other words, by the time gap between the moment when the order for stimulation is issued and the moment when the stimulation is actually conveyed.

The stimulation time refers to the period of time in which the suction- or puff-type pressure is provided. Two three-way solenoid valves are individually controlled to be $\mathrm{ON}$ or OFF with a temporal resolution of approximately $0.1 \mathrm{~s}$. The time during which stimulation is not provided is defined as the air discharge mode (Table 1).

Table 1 Four modes of air movement in the air stimulation system, based on the statuses of the valves

\begin{tabular}{llll}
\hline Mode & Valve 1 & Valve 2 & Intake Valve \\
\hline Suction stimulus & OFF & ON & OFF \\
Air discharge & ON & ON & OFF \\
Puff stimulus & Doesn't Matter & OFF & OFF \\
Air intake & ON & ON & ON \\
\hline
\end{tabular}

The character liquid crystal display of the control unit and four switches is used to manually control the type, intensity, and duration of the stimulation. When using a PC, the generation of control signals can be controlled by the E-Prime software. The stimulation parameter information and stimulation trigger signals can be transmitted through a USB connector.

\section{Air-handling unit}

The air-handling unit is composed of an air compressor S3040-3 (SeoWon Compressor, South Korea), two three-way solenoid valves, an air pressure regulator, and an air tube. The flow rate of the air compressor is $264 \mathrm{~L} / \mathrm{min}$, and the maximum working pressure is $123 \mathrm{psi}$. The dimensions of the air compressor are $74 \times 32 \times 67 \mathrm{~cm}^{3}(\mathrm{~W} \times \mathrm{L} \times \mathrm{H})$, with a weight of $35.4 \mathrm{~kg}$. Each three-way solenoid valve has a left, middle, and right fluid connection port arranged in a $\mathrm{T}$ shape. The ports change the direction of fluid flow. Depending on the control signal, a single input port is connected to one of two output ports. The HDW022T-3 model has its middle port open all the time, and depending on the control signal, it is connected to either the left port (OFF) or the right port $(\mathrm{ON})$. The HDW022G-3 model has its right port always open and, depending on the control signal, it is connected to either the left port $(\mathrm{OFF})$ or the middle port $(\mathrm{ON})$.

The air pressure regulator is composed of a ball valve BVB-F-04T (Hohwon Co., South Korea) and stepper motor 42HS40-1704A05 (MAKEZONE Co., South Korea). The handle of the ball valve was removed, and a gear was used to provide a mechanical connection to the stepper motor (Fig. 2). The rotation angle of the stepper motor was controlled to adjust the cross-section through which the compressed air passes. When the ball valve is "opened" and its cross-section becomes the same as that of the air tube, maximum pressure is delivered. When the cross-section becomes small, low pressure is provided. All components have 12 and $8 \mathrm{~mm}$ as their external and internal diameters, respectively, and are connected with urethane air tubes.

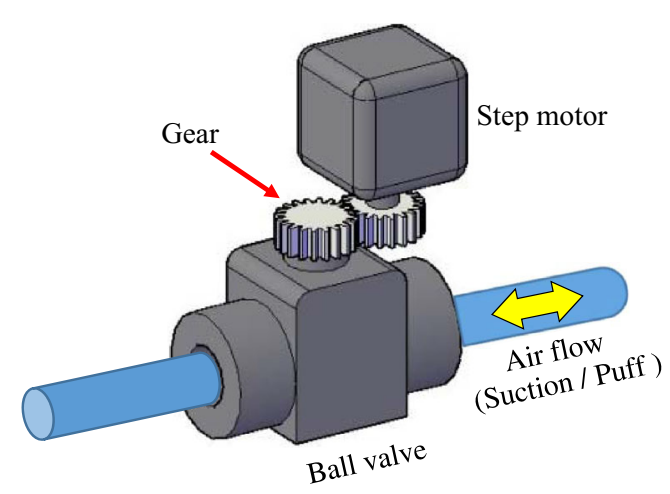

Fig. 2 Schematic of the air pressure regulator 


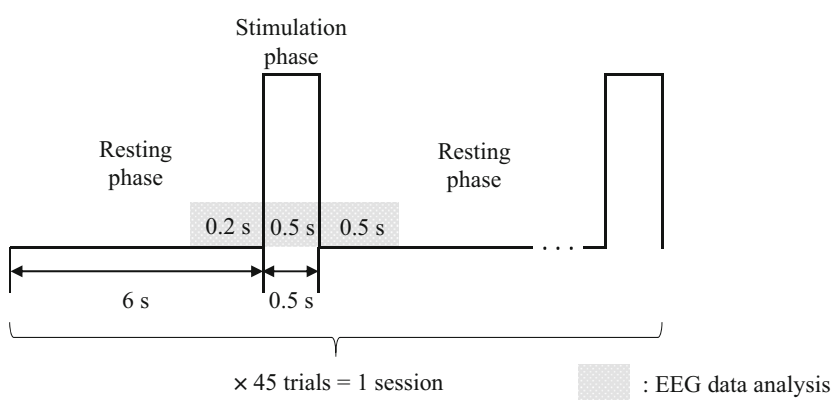

Fig. 3 Experimental design

\section{Stimulation unit}

The stimulation unit was designed to provide pressure to a surface area using the same size and position of a finger (Fig. 1). A two- and three-dimensional computer-aided design program, AutoCAD (Autodesk, USA), was used to create its design, and the Mojo 3-D printing system (Stratasys, USA) was used to 3-D-print the prototype. The area of stimulation was set as $6 \times 6 \mathrm{~mm}^{2}$, based on the size of a finger and of the air tube connecting it to the air-handling unit.

\section{Validation}

The first joint of the right index finger is laid on the simulation unit while applying the suction- or puff-type pressure at two levels ( 2 or $6 \mathrm{psi})$ for $0.5 \mathrm{~s}$. To verify the performance of the system, the output signal of the pressure sensor was recorded by a digital phosphor oscilloscope TDS3034 (Tektronix, USA), and analyzed by MATLAB (MathWorks Inc., USA).

In addition, a preliminary electroencephalographic (EEG) experiment was conducted. The participants were ten adult males $(23.9 \pm 2.6$ years old $)$ and ten adult females $(21.7 \pm$ 1.2 years old). Before the experiment, we requested that participants not use tobacco, caffeine, drugs, or alcohol for one day prior to the experiment because these substances are known to affect the central nervous system. On the day of the experiment, we verified that every participant prepared for the experiment with this restriction, and excluded the ones who failed to do so. None of the participants reported a history of psychiatric or neurologic disorders, and all subjects were right-handed as a result of the revised Edinburgh Reading Test (Oldfield, 1971), which gave consent for the procedure. The brainwaves were recorded using Quick Amp (Brain Products, Germany). The somatosensory area $\mathrm{C} 3$ and another 15 positions (AF3/4, F3/4/z, FC1/2/5/6, C3/4/z, CP1/2, Pz, Oz) were monitored with $\mathrm{Ag} / \mathrm{AgCl}$ electrodes placed according to the 10-20 electrode system of the International Federation of Societies for Electroencephalography and Clinical Neurophysiology. The sampling rate was $500 \mathrm{~Hz}$.

Each test participant was directed to lay his or her right arm on the desk where the stimulation unit was positioned, while sitting comfortably in a chair. Body movement was limited as much as possible before test start to stabilize the brain. Since an air compressor generates significant noise, it could cause auditory contamination in brain waves, so the compressor was located in a space separated by two sound insulation walls from the experimental space containing the test participants. The distance between the two locations was $7 \mathrm{~m}$. The sound noise level was measured by a digital sound level meter DL1351 (Leaton, USA) near the test participants and at the location of the air compressor. The values were 37.2 and 79.8 $\mathrm{dBA}$, respectively. This result confirms that the sound of the air compressor was successfully blocked. A black curtain was used to block visual stimulation. The protocol for the research project was approved by the Institutional Review Committee of Konkuk University, in which the research was undertaken, and the study conformed to the provisions of the Declaration of Helsinki.

A single trial of the experiment comprised the resting phase $(6 \mathrm{~s})$ and the stimulation phase $(0.5 \mathrm{~s})$ (Fig. 3). The resting phase was a standby phase that occurred while the fingers lay on the stimulation unit, which prevented memory effects. In

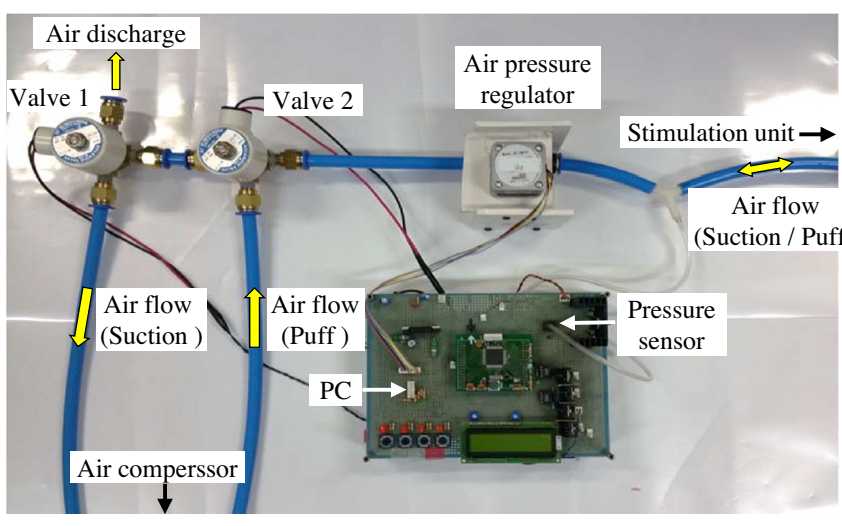

(a)

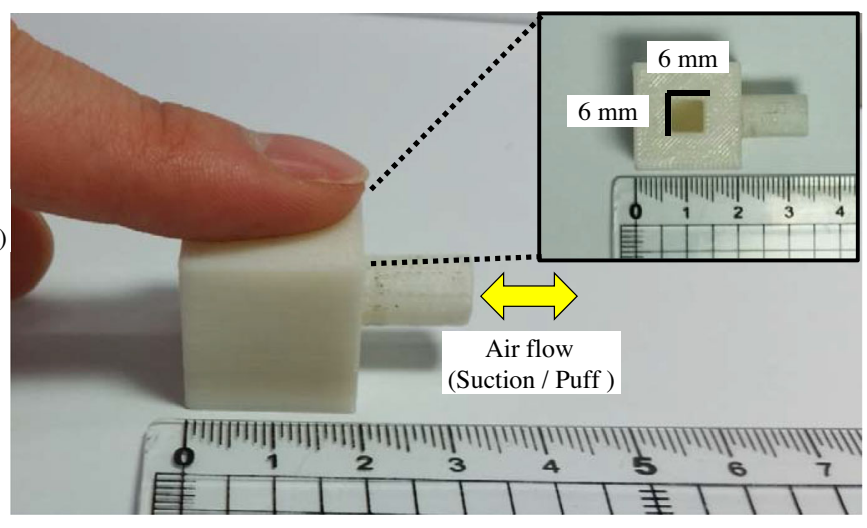

(b)

Fig. 4 Photograph of (a) the developed stimulation system and (b) a view of stimulation unit 


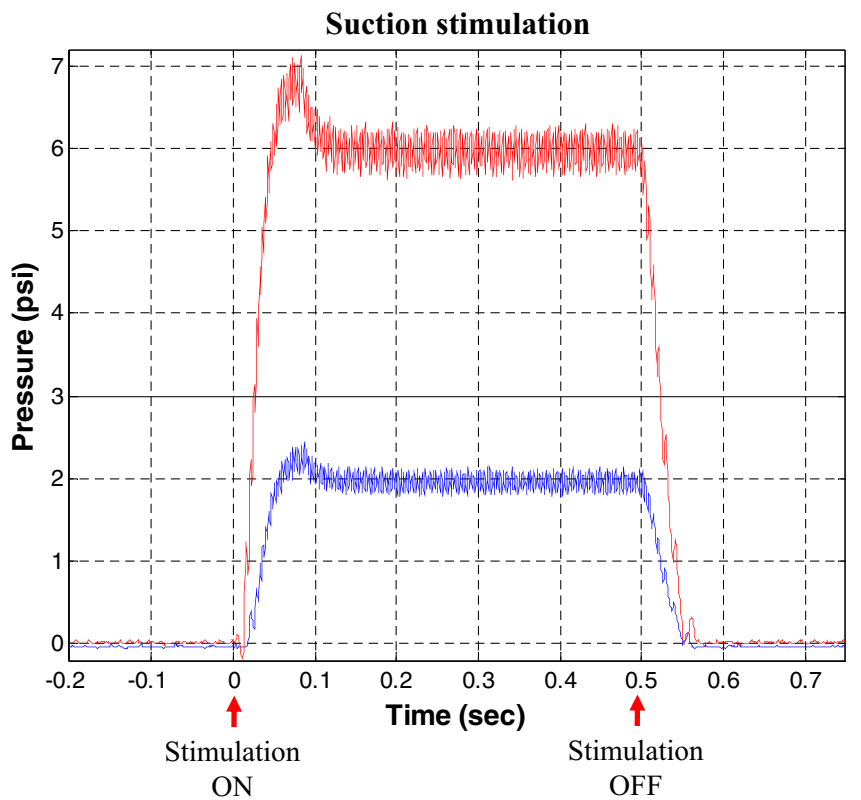

(a)

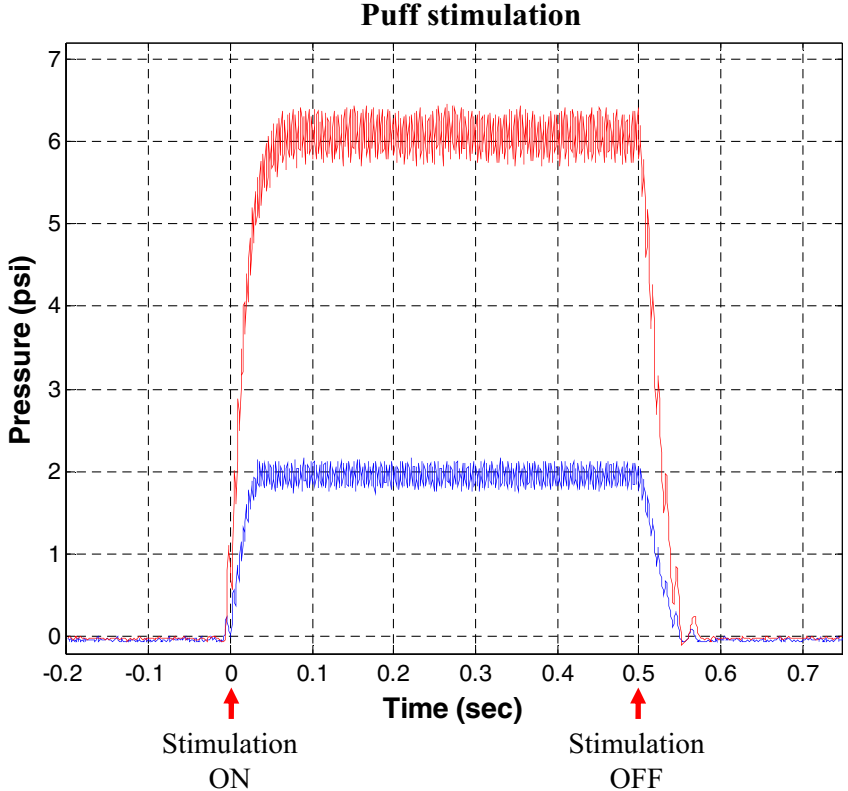

(b)

Fig. 5 Raw data from the pressure sensor, showing (a) suction and (b) puff stimulation at two intensity levels (2 and 6 psi)

the stimulation phase, one of two intensities ( 2 or 6 psi) with one of two pressure types or a sham stimulation was administered, summing to a total of five possible types of stimulation. The five stimulation types were administered in random order, nine times each; this was defined as a single session. Therefore, a single session included 45 trials in total ( 5 types $\times$ 9 times $=45$ trials), and two such sessions were administered to each participant.

MATLAB (MathWorks, USA) was used to analyze the brain waves. The raw brain-wave data were band-passfiltered between 0.5 and $20 \mathrm{~Hz}$. Three experimental epochs were defined for a brain-wave analysis: $0.2 \mathrm{~s}$ prior to stimulation, the $0.5 \mathrm{~s}$ during stimulation, and $0.5 \mathrm{~s}$ after stimulation (Fig. 3). First, C3, which was the somatosensory area contralateral to the simulation site (right finger), was analyzed using a mean event-related potential (ERP) function for each subject in order to extract the peak-to-peak and slope values. The peak-to-peak value was the difference between the maximum positive peak and the minimum negative peak voltages, and the slope was the gradient of a line drawn between the two peaks. To determine whether the extracted peak-to-peak values and the value of the slope show significant difference

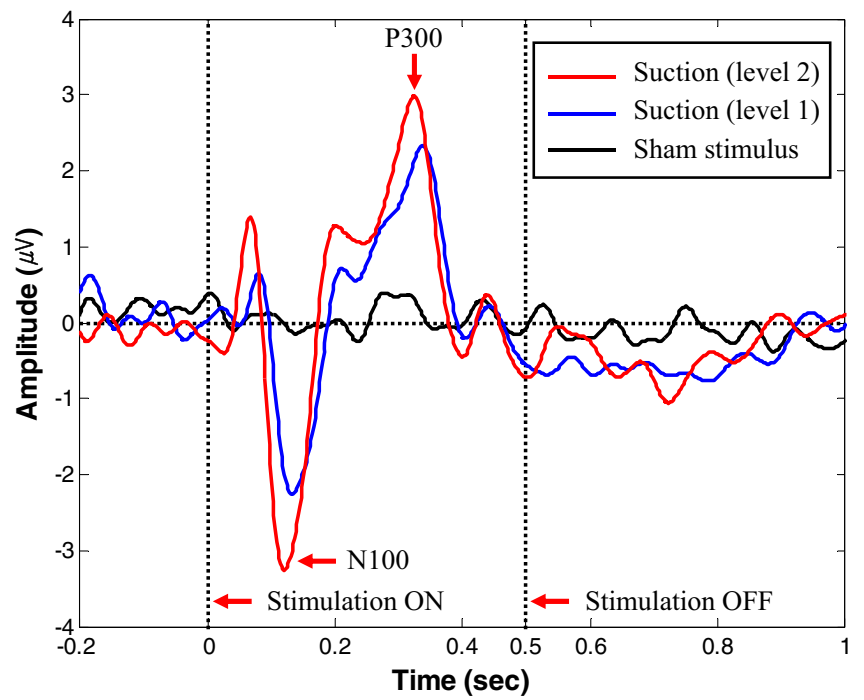

(a)

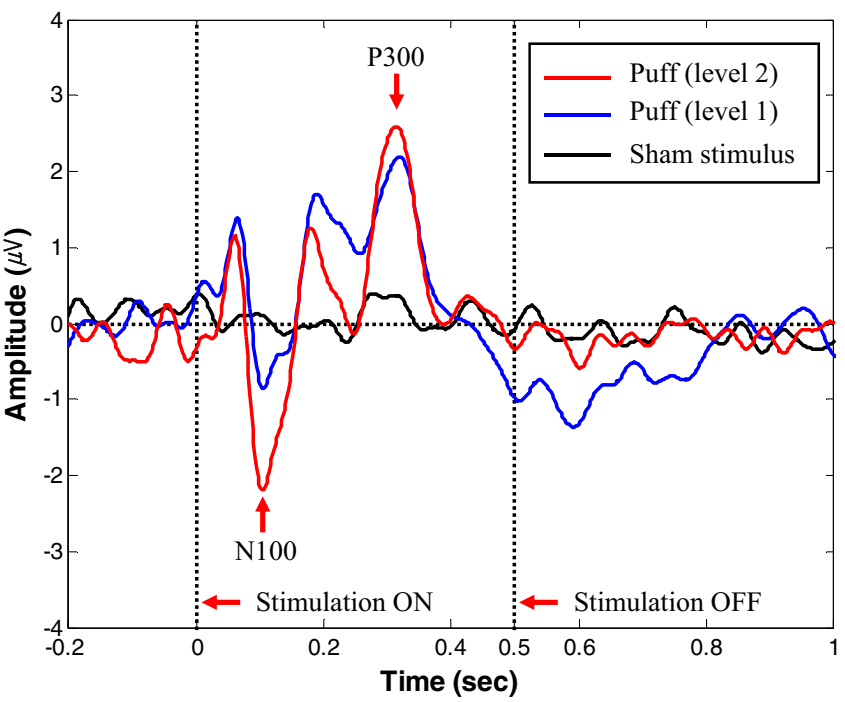

(b)

Fig. 6 Mean event-related potentials at standard electrode position C3 elicited by (a) suction and (b) puff stimulation at two intensities 
Table 2 Means, standard deviations, and statistical results of peak-to-peak values $(\mu \mathrm{V})$ and peak-to-peak slopes $(\mu \mathrm{V} / \mathrm{s})$ for event-related potentials at scalp electrode $\mathrm{C} 3$ for suction and puff stimulation, each at two intensity levels ( 2 and 6 psi)

\begin{tabular}{|c|c|c|c|c|c|}
\hline & \multicolumn{2}{|c|}{ Suction } & \multicolumn{2}{|c|}{ Puff } & \multirow{2}{*}{$\begin{array}{c}\text { two-way repeate } \\
\text { ANOVA }\end{array}$} \\
\hline & Level 1 & Level 2 & Level 1 & Level 2 & \\
\hline $\begin{array}{c}\text { Peak-to-Peak } \\
(\mu \mathrm{V}) \\
(\text { Mean } \pm \text { Std })\end{array}$ & $\underset{7.70 \pm 3.27}{\Gamma}$ & $* \frac{*}{9.12 \pm 3.37}$ & $7.40 \pm 2.01$ & $8.22 \pm 2.65$ & $\begin{array}{c}\text { Intensity: } p=.001 \\
\text { Type: } p=.199\end{array}$ \\
\hline $\begin{array}{c}\text { Slope }_{\text {P-P }} \\
(\mu \mathrm{V} / \mathrm{sec}) \\
(\text { Mean } \pm \text { Std })\end{array}$ & $\begin{array}{r}\Gamma^{\top} * \\
25.34 \pm 3.42 \\
亡\end{array}$ & $\begin{array}{r}*-1 \\
32.00 \pm 4.11 \\
\\
2 * * * \\
\end{array}$ & $\begin{array}{r}\Gamma_{14.38 \pm 2.04} * \\
\end{array}$ & $* \underbrace{}_{23.01 \pm 3.75}$ & $\begin{array}{c}\text { Intensity: } \mathrm{p}<.001 \\
\text { Type: }<<.001\end{array}$ \\
\hline
\end{tabular}

for each stimulation type (suction and puff) and stimulation intensity (Level 1 and 2), a two-way repeated analysis of variance (ANOVA; PASW Statics 18, Hong Kong) was conducted. In addition, a paired $t$ test (PASW Statics 18, Hong Kong) was carried out on the variables (type or intensity of stimulation) that showed significant differences. Regarding the entire brain area, a time-varying topographic map was used to observe changes in the ERP signal. At each time point, the ERP data averaged across participants were subtracted from the mean of the baseline signal at the start of stimulation.

\section{Results}

Photographs of the realized system are shown in Fig. 4. The overall dimensions of the stimulation unit are $20 \times 32 \times 20$
$\mathrm{mm}^{3}(\mathrm{~W} \times \mathrm{L} \times \mathrm{H})$, with a weight of $23 \mathrm{~g}$. We confirmed that the system works stably.

The raw data of the two types of pressure stimulation as measured by the pressure sensor are shown in Fig. 5 . Although there was an initial overshoot, the predetermined suction pressure was successfully reached within approximately $0.1 \mathrm{~s}$ (Fig. 5a), and within $0.05 \mathrm{~s}$ during puff-type pressure stimulation (Fig. 5b). Both stimulations returned to baseline within $0.05 \mathrm{~s}$ after the end of stimulation. In addition, for both types of stimulation, the desired final pressure was accurately held during the period of stimulation.

At electrographic position $\mathrm{C} 3$, representing somatosensory cortex, the mean ERP signal change is shown in Fig. 6 for all conditions tested. Except in the sham case, clear ERP signal changes are confirmed through both the N100 and P300. Table 2 lists the means, standard deviations, and statistical results

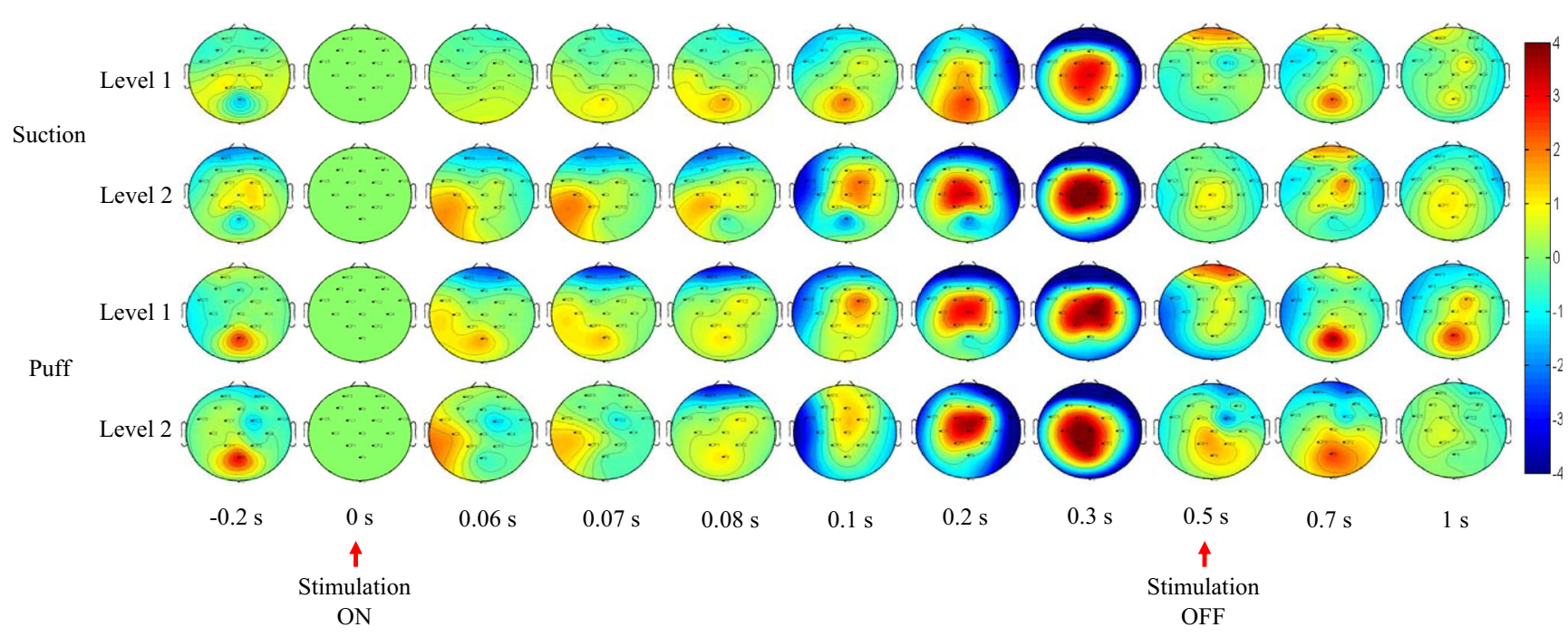

Fig. 7 Time-varying topographic maps of entire brain responses to the suction and puff stimulation, each at two intensity levels. The labeled dots are standard 10-20 scalp electrode positions. The colored bar at right is calibrated in microvolts 
for the peak-to-peak values $(\mu \mathrm{V})$ and peak-to-peak slopes $(\mu \mathrm{V} / \mathrm{s})$. For peak-to-peak values, only the stimulation intensity showed a significant difference $(p=.001)$, and the stimulation type did not $(p=.199)$. The results of a paired $t$ test on stimulation intensity displayed a significant difference only between the Suction 1 and Suction 2 levels $(p<.001)$. For the slopes, significant differences were displayed for both stimulation intensity $(p<.001)$ and stimulation type $(p<.001)$. The results of a paired $t$ test for both types and intensities of stimulation demonstrated that the value of the slope increased following an increase in both suction stimulation and puff stimulation (suction/puff: $p s<.001$ ). At the same stimulation intensity, the value of the slope was higher for suction than for puff stimulation (Level 1/Level 2: $p$ s <.001).

Figure 7 shows the time-varying topographic maps of the mean ERP signals for suction and puff stimulations with two levels of intensity each. At approximately $0.1 \mathrm{~s}$ after the application of stimulation, the contralateral somatosensory area C3 shows an N100. At approximately $0.3 \mathrm{~s}$, the electrodes in and around the somatosensory association area $(\mathrm{C} 1, \mathrm{FC} 1, \mathrm{Cz}$, and $\mathrm{CP} 1$ ) all show positive-polarity peaks, including $\mathrm{C} 3$, which shows a P300. At approximately $0.7-1 \mathrm{~s}$, the stimulus returns to baseline.

\section{Discussion}

The tactile stimulator developed in this study is composed of three parts: a control unit, air-handling unit, and stimulation unit. The stimulator is simple in structure and is capable of providing both puff and suction types of stimulus by using a single air compressor. In addition, since it uses only off-the-shelf parts, manufacturing the stimulator is easy and inexpensive.

By suitably programming the microcontroller, the type, intensity, and duration of the stimulation can be controlled. The stimulation type can be selected by opening and closing the valves, and the flow direction of the fluid can be selected. Although some fluctuations are present, the intensity of the stimulation can be varied in steps of 1 psi within a range of $2-$ 6 psi. In a system performance test, the maximum rise and minimum fall times were, respectively, 0.1 and $0.05 \mathrm{~s}$. Thus, in the case of repetitive (discontinuous) pressure stimulation, the maximum repetition rate can be provided up to $5 \mathrm{~Hz}$, since the minimum possible period is $0.2 \mathrm{~s}$ from these data. In the case of sustained (continuous) stimulation, the stimulation period can be precisely controlled to $0.1 \mathrm{~s}$. The air-handling unit delivers power quantitatively to the stimulation unit, on the basis of a simple structure consisting of air compressor, threeway solenoid valve, air pressure regulator, and air tube.

Generally, in studies of the tactile sense, the experiment must be conducted repetitively to reach significance. Therefore, the stimulation unit should be manufactured using rigid material to ensure stationary statistics. In this study, a material of superior mechanical properties (tensile strength of 4,700 psi, flexural strength of $8,450 \mathrm{psi}$, and deflection temperature of $96^{\circ} \mathrm{C}$ ) was used to print the tactor using 3-D printing technology. Therefore, the pressure applied by the test participant, the force applied by the air compressor, external physical forces along two directions, and softening caused by the warmth of the skin are not expected to influence the results of these experiments.

Puff and suction pressures were delivered to the right index finger at two different intensities to elicit a mean ERP pattern from the somatosensory area (C3) as well as time-varying topographic maps of the entire brain. The results showed that $\mathrm{N} 100$, which is related to the perception of the tactile sense, and P300, which occurs during the brain's data processing procedure and is related to cognition following external stimulation, were both well developed. This proves that the system can be used for studying the tactile sense with regard to the puff- and suction-type pressures. Although the results are preliminary, we found that there is a difference in the peak-topeak and slope measurements between the two different types of stimulation and between the two different intensities. This also means that there is a difference in the reactions of the mechanoreceptors depending on the type and intensity of the stimulation, which is subsequently reflected in the gross data processing patterns of the brain.

\section{Conclusion}

In this study, a tactile stimulator capable of delivering both puff- and suction-type stimuli was developed. A performance evaluation of the system demonstrated that quantitative control of the stimulation parameters is possible. A preliminary experiment with 20 humans verified the utility of the system for objective perceptual studies. The system will be actively used for comparative puff- and suction-type pressure studies on a human level. Further studies on the use of this system for analyzing brain information processing according to the two types of pressure sensitive stimuli and various intensities will provide base data that can be applied in clinical tests and NUIs. Although only puff-type stimulation has been used to diagnose neurological disorders, such as Parkinson's disease and chronic pain, and to examine syndromes related to tactile nerves, such as tactile extinction and tactile hallucination, this system can provide data that can be utilized to objectively determine the clinical utility and effectiveness of the suctiontype stimulation method. In addition, further research on the use of this system can be conducted to provide evidence of brain neurology that could be used to expand the suction-type stimulation method, whose application has been limited as compared to the puff-type stimulation method.

Author note This work was supported by a Mid-Career Researcher Program Grant through the National Research Foundation of Korea (NRF), funded by the Ministry of Education (MOE) (No. 2017R1A2B2004629). Also supported by the Pioneer Research Center 
Program through the National Research Foundation of Korea, funded by the Ministry of Science, ICT \& Future Planning (2011-0027920).

\section{References}

Allen, H. A., \& Humphreys, G. W. (2009). Direct tactile stimulation of dorsal occipito-temporal cortex in a visual agnostic. Current Biology, 19, 1044-1049. doi:10.1016/j.cub.2009.04.057

Auvray, M., Gallace, A., Hartcher-O’Brien, J., Tan, H. Z., \& Spence, C. (2008). Tactile and visual distractors induce change blindness for tactile stimuli presented on the fingertips. Brain Research, 1213, 111-119. doi:10.1016/j.brainres.2008.03.015

Bagriantsev, S. N., Gracheva, E. O., \& Gallagher, P. G. (2014). Piezo proteins: Regulators of mechanosensation and other cellular processes. Journal of Biological Chemistry, 289, 31673-31681.

Bark, K., McMahan, W., Remington, A., Gewirtz, J., Wedmid, A., Lee, D. I., \& Kuchenbecker, K. J. (2013). In vivo validation of a system for haptic feedback of tool vibrations in robotic surgery. Surgical Endoscopy, 27, 656-664. doi:10.1007/s00464-012-2452-8

Chouvardas, V. G., Miliou, A. N., \& Hatalis, M. K. (2008). Tactile displays: Overview and recent advances. Displays, 29, 185-194.

Dresel, C., Parzinger, A., Rimpau, C., Zimmer, C., Ceballos-Baumann, A. O., \& Haslinger, B. (2008). A new device for tactile stimulation during fMRI. Neurolmage, 39, 1094-1103.

Echenique, A. M., \& Graffigna, J. P. (2011). Electrical stimulation of mechanoreceptors. Journal of Physics: Conference Series, 332, 012044.

Gallace, A., Tan, H. Z., Haggard, P., \& Spence, C. (2007). Short term memory for tactile stimuli. Brain Research, 1190, 132142. doi:10.1016/j.brainres.2007.11.014

Gallasch, E., Fend, M., Rafolt, D., Nardone, R., Kunz, A., Kronbichler, M., ..., \& Golaszewski, S. (2010). Cuff-type pneumatic stimulator for studying somatosensory evoked responses with fMRI. NeuroImage, 50, 1067-1073. doi:10.1016/j.neuroimage.2010.01.014

Golaszewski, S. M., Zschiegner, F., Siedentopf, C. M., Unterrainer, J., Sweeney, R. A., Eisnerf, W.,..., \& Felver, S. (2002). A new pneumatic vibrator for functional magnetic resonance imaging of the human sensorimotor cortex. Neuroscience Letters, 324, 125-128.

Gwilliam, J. C., Bianchi, M., Su, L. K., \& Okamura, A. M. (2013). Characterization and psychophysical studies of an air-jet lump display. IEEE Transactions on Haptics, 6, 156-166.

Ho, C., Santangelo, V., \& Spence, C. (2009). Multisensory warning signals: When spatial correspondence matters. Experimental Brain Research, 195, 261-272. doi:10.1007/s00221-009-1778-5

Jousmaki, V., Nishitani, N., \& Hari, R. (2007). A brush stimulator for functional brain imaging. Clinical Neurophysiology, 118, 2620 2624

Kaczmarek, K. A., Nammi, K., Agarwal, A. K., Tyler, M. E., Haase, S. J., \& Beebe, D. J. (2006). Polarity effect in electrovibration for tactile display. IEEE Transactions on Bio-medical Engineering, 53, 2047 2054.

Kim, H. S., Choi, M. H., Kim, H. J., Hong, S. P., Park, J. Y., Jun, J. H., ..., \& Chung, S. C. (2014). Development of a simple pressure and heat stimulator for intra- and inter-digit functional magnetic resonance imaging. Behavior Research Methods, 46, 396-405. doi:10.3758/ s13428-013-0371-9
King, C. H., Franco, M., Culjat, M. O., Higa, A. T., Bisley, J. W., Dutson, E., \& Grundfest, W. S. (2008). Fabrication and characterization of a balloon actuator array for haptic feedback in robotic surgery. Journal of Medical Device, 2, 041006.

Makino, Y., \& Shinoda, H. (2005). Selective stimulation to superficial mechanoreceptors by temporal control of suction pressure. In First Joint Eurohaptics Conference and Symposium on Haptic Interfaces for Virtual Environment and Teleoperator Systems. World Haptics Conference (pp. 229-234). Piscataway, NJ: IEEE Press.

Martinac, B. (2004). Mechanosensitive ion channels: Molecules of mechanotransduction. Journal of Cell Science, 117, 2449-2460.

Monnai, Y., Hasegawa, K., Fujiwara, M., Yoshino, K., Inoue, S., \& Shinoda, H. (2014). HaptoMime: Mid-air haptic interaction with a floating virtual screen. In Proceedings of the 27th annual ACM symposium on User interface software and technology (pp. 663667). New York: ACM.

Oldfield, R. C. (1971). The assessment and analysis of handedness: The Edinburgh Inventory. Neuropsychologia, 9, 97-113. doi:10.1016/ 0028-3932(71)90067-4

Philip, P. H., Sodergren, M. H., Lord, A. C., Teare, J., Yang, G. Z., \& Darzi, A. (2015). Consumer demand for surgical innovation: A systematic review of public perception of NOTES. Surgical Endoscopy, 29, 774-780.

Porquis, L. B. C., Konyo, M., \& Tadokoro, S. (2012). Tactile-based torque illusion controlled by strain distributions on multi-finger contact. In 2012 I.E. Haptics Symposium (HAPTICS) (pp. 393-398). Piscataway: IEEE Press.

Ritter, P., Moosmann, M., \& Villringer, A. (2009). Rolandic alpha and beta EEG rhythms' strengths are inversely related to fMRI-BOLD signal in primary somatosensory and motor cortex. Human Brain Mapping, 30, 1168-1187.

Skedung, L., Arvidsson, M., Chung, J. Y., Stafford, C. M., Berglund, B., \& Rutland, M. W. (2013). Feeling small: Exploring the tactile perception limits. Scientific Reports, 3, 2617.

Suzuki, Y., \& Kobayashi, M. (2005). Air jet driven force feedback in virtual reality. IEEE Computer Graphics and Applications, 25, 44-47.

Tsalamlal, M. Y., Ouarti, N., \& Ammi, M. (2013). Psychophysical study of air jet based tactile stimulation. In World Haptics Conference (WHC), 2013 (pp. 639-644). Piscataway: IEEE Press.

Wall, S. A., \& Brewster, S. (2006). Sensory substitution using tactile pin arrays: Human factors, technology and applications. Signal Processing, 86, 3674-3695.

Yamamoto, A., Nagasawa, S., Yamamoto, H., \& Higuchi, T. (2006). Electrostatic tactile display with thin film slider and its application to tactile telepresentation systems. IEEE Transactions on Visualization and Computer Graphics, 12, 168-177.

Yang, P. F., Wang, F., \& Chen, L. M. (2015). Differential fMRI activation patterns to noxious neat and tactile stimuli in the primate spinal cord. Journal of Neuroscience, 35, 10493-10502. doi:10.1523/ JNEUROSCI.0583-15.2015

Yuan, H., Liu, T., Szarkowski, R., Rios, C., Ashe, J., \& He, B. (2010). Negative covariation between task-related responses in alpha/betaband activity and BOLD in human sensorimotor cortex: An EEG and fMRI study of motor imagery and movements. Neurolmage, 49, 2596-2606. doi:10.1016/j.neuroimage.2009.10.028 